\title{
Fractal Rain Distributions and Chaotic Advection
}

\author{
Ronald Dickman \\ Departamento de Física, ICEx, Universidade Federal de Minas Gerais, \\ Caixa Postal 702, 30161-970 Belo Horizonte, Minas Gerais, Brazil
}

Received on 1st December, 2003

\begin{abstract}
Localized rain events have been found to follow power-law distributions over several decades, suggesting parallels between precipitation and seismic activity [O. Peters et al., PRL 88, 018701 (2002)]. Similar power laws can be generated by treating raindrops as passive tracers advected by the velocity field of a two-dimensional system of point vortices [R. Dickman, PRL 90, 108701 (2003)]. Here I review observational and theoretical aspects of fractal rain distributions and chaotic advection, and present new results on tracer distributions in the vortex model.
\end{abstract}

\section{Introduction}

Complex systems often exhibit fractal or power-law scaling; Earth's atmosphere is no exception. Fractal rain distributions have been known for at least two decades [1, 2, 3], while recent analyses indicate that durations of dry intervals, and the size of rain events, follow power laws [4, 5]. The similarity between the latter observations and scaling laws in seismic activity suggests a parallel between rain and earthquakes, and a possible connection with the phenomenon of self-organized criticality [5, 6].

Atmospheric motion is turbulent, particularly in the vicinity of storms, and various aspects of turbulent flow follow power laws over many orders of magnitude [7, 8, 9]. Even in the absence of fully developed turbulence, unsteady flow may stretch and fold an initially compact region, leading to a highly convoluted, nonuniform density of suspended particles or droplets $[10,11,12]$ via chaotic advection $[13,14]$. In light of these observations, it is interesting to develop a model in which rain is an ideal passive tracer $[15,16]$. In [17] it was shown that such a model is capable of producing power-law-distributed event sizes and durations.

In this paper I review some of the evidence for fractal rain distributions, and present new results on the spatial distribution of tracers in the vortex model. Progress in fluid mechanics depends heavily on numerical solution of the equations of motion, which in turn represents one of the most challenging areas in computational physics, the theme of the present number.

In Sec. II, I survey observations of fractal rain distributions. Sec. III contains a brief discussion of SOC-like approaches, while Sec. IV reviews results on tracer-particle dynamics in a fluid undergoing chaotic advection. I define the vortex model in Sec. V, which also includes a summary of previous findings and some recent extensions. Sec. VI presents new results on spatial distributions of tracers in the vortex model. The paper closes in Sec. VII with a summary and discussion of open questions.

\section{Fractal rain distributions}

Discussions of fractal rain distributions go back at least to the work of Lovejoy and Mandelbrot [1] who presented a model with a single fractal dimension. The distribution in question involves a time series of duration $T$ and a fixed observation point or station. The observation interval is partitioned into $N=T / \tau$ subintervals of duration $\tau$, each characterized as rainy (a nonzero amount of rain is detected at the station in this interval) or dry. The function $r(\tau)$ is then defined as the number of rainy subintervals at scale $\tau$. Olsson et al. found that this distribution follows a power law, $r \sim \tau^{-\gamma}$, with $\gamma \simeq 0.8$, over a certain range of durations [2]. Note that for $\tau \approx T, r \rightarrow N$ (all subintervals are rainy), while for $\tau$ much shorter than the characteristic time between raindrops, $r$ saturates at a value $M$ equal to the total number of raindrops incident on the station during the interval $T$. Between these simple limits, $r(\tau)$ may exhibit nontrivial behavior reflecting correlations in the generation or dynamics of raindrops. Now, if the arrival times of the raindrops were mutually independent (so that the time interval between successive drops at the detector were exponentially distributed), the number of drops $n(\tau)$ in a given subinterval would be Poisson-distributed with mean $\langle n(\tau)\rangle=m \tau$, with $m=M / T$, and we would have $r(\tau)=(T / \tau)\left(1-e^{-m \tau}\right)$. Thus a power law distribution with $\gamma<1$ rules out a simple "independent event" model, suggesting some nonlinear mechanism behind the observed rainfall statistics.

The observations of Olsson et al. (from Sweden) were later corroborated by Lavergnat and Golé [3] in an experiment performed near Paris. The latter study generated data on raindrop arrival times and sizes over a 14-month period, and confirmed the scaling $r \sim \tau^{-0.82}$ over six orders of magnitude (from 0.01 to $10^{4}$ minutes). Other important conclusions from this study are: (1) the raindrop diameter distribution decays roughly exponentially (or perhaps as a stretched exponential) for diameters greater than about 0.5 
$\mathrm{mm}$; (2) the distribution of time intervals between raindrops can be fit to a so-called bi-Pareto distribution over about nine orders of magnitude. This distribution involves two power law regimes, one for short times (drops associated with a given storm) another for long times (intervals between successive storms). On the basis of their analysis Lavergnat and Gole conclude that the waiting time $D$ between successive rain events is power-law distributed: $P_{d}(D) \sim D^{-\tau_{D}}$ with $\tau_{D}=1.68$. (For $D \approx$ one day the probability density $P_{d}$ decays rapidly; droughts longer than a week or so were not seen in their experiment.)

Convincing evidence for a multifractal spatial distribution of raindrops in storms, on scale from $1 \mathrm{~cm}$ up to meters, was very recently reported by Lovejoy et al. [18]. An important conclusion of these authors is that there is no meaningful way to describe rain content in the atmosphere in terms of a smoothly varying density, since large fluctuations are present at all scales. The authors suggest turbulence as the reason for the fluctuations in raindrop distribution.

Recently a large time-series (six months) from radar observations on the Baltic coast became available under the BALTEX project [19]. The radar station determines the quantity of rain falling in a $1 \mathrm{~m}^{2}$ column of the atmosphere. Arrival times of individual raindrops are not resolved, but the total amount of rain above the station at each $1 \mathrm{~min}$. interval is registered. The threshold for detection is 0.005 $\mathrm{mm} / \mathrm{h}$; intervals with a precipitation rate above this threshold have a nonzero rate $q(t)$, otherwise $q(t)=0$ for that interval. In their analysis of the BALTEX data, Peters et al. focus on rain events, defined as sequences of consecutive intervals with nonzero rainfall $[4,5]$. A series of consecutive intervals having zero rain defines a drought. The intensity $I=\sum_{t} q(t)$ of a rain event is the rainfall integrated over its duration. Peters, Hertlein and Christensen found that the distribution of rain-event sizes at the Baltic coast station follows a power law over at least three decades. Drought durations are also power-law distributed over the range of several minutes to about a week, with a significant perturbation apparently reflecting diurnal variation. The power laws identified by Peters et al. may be expressed in the form

$$
P_{i}(I) \sim I^{-\tau_{I}}
$$

and

$$
P_{d}(D) \sim D^{-\tau_{D}}
$$

where $P_{i}$ and $P_{d}$ are the probability distributions for rain event intensities, and for drought durations, and the exponents are found to take the values

$$
\tau_{I}=1.36 \quad \tau_{D}=1.42
$$

These authors emphasize the similarities between these distributions and those found for earthquakes, suggesting a parallel with self-organized criticality to be discussed in the following section.

Taken as a whole, the observations of Olsson et al., Lavergnat and Golé, Lovejoy and co-workers, and Peters et al. present a very strong case for fractal or multifractal distributions of rain at a given position over time, and in space, at a given instant [20]. The universality of the observed distributions is less clear. First, the time series all come from the north of Western Europe, where prolonged dry periods are evidently rare. The central region of Minas Gerais, Brazil (to cite one example) experiences a dry spell of several months each year, and might therefore exhibit a different distribution of droughts. The Paris and Sweden experiments yielded similar values $(\gamma=0.82)$ for the exponent characterizing the fractal distribution in time, while the BALTEX data yield $\gamma \simeq 0.55$ [5]. On the other hand, the Paris results suggest $\tau_{D}=1.68$, considerably larger than the Baltic observations. Observations from other sites (in particular, from other regions of the world, including continental sites, and oceans), are needed to confirm the generality of power laws, and the range of exponent values.

\section{Rain and Self-organized criticality}

Peters, Hertlein and Christensen noted a striking similarity between the scaling laws they found in the rain data and those known for earthquakes. Specifically, earthquake magnitudes $M$ (defined in terms of energy released) follow the Gutenberg-Richter law $P_{m}(M) \sim M^{-\tau_{M}}$ [21], while the waiting time between earthquakes in a given region follows a power-law known as Omori's law [22, 23]. This suggests a parallel between precipitation in the atmosphere and relaxation of the Earth's crust at stressed tectonic-plate boundaries [5]. In the context of seismology, cooperative relaxation due to elastic interactions and nonlinear friction is captured by block-spring models $[24,25]$ or, in muchreduced fashion, by sandpile models [6]. The latter have attracted much attention as the principal example of the selforganized criticality paradigm for scale-invariance in natural, far-from-equilibrium systems [6, 26, 27].

Indeed, sandpile-like models of rainfall have been studied [28, 29]. They involve the directed motion of raindrops such that when a given cell contains more than a certain number of drops, the latter move to cells at the level below. That such a model yields power-law distributions for sizes of certain kinds of events is not surprising, as this is an intrinsic feature of sandpile models [26, 30]. (It is less clear how to define the duration of a rain event, since sandpiles represent a singular limit in which event durations cannot be measured on the same time scale as intervals between events $[31,32]$.)

But if certain aspects of rain distributions resemble those of avalanches in sandpile-like models, the underlying physics remains obscure. While it may yet prove possible to explain the observed power laws in terms of an open, driven dissipative system [28, 29, 33], there is no obvious reason for the formation or precipitation of one raindrop to provoke similar events nearby. Given the attendant release of latent heat, one might instead expect a self-limiting tendency in condensation.

In fact, condensation and precipitation of rain is a complex process, involving the interplay between atmospheric motion, including turbulent convection, thermodynamics and nucleation processes [34]. Evaporation, condensation and vertical fluid motion are strongly coupled via buoyancy. 
While it is hard to see how direct interactions between raindrops over a mean interparticle distance of $10 \mathrm{~cm} \mathrm{[18]} \mathrm{could}$ lead to clustering, the drops are of course highly influenced by the motion of the surrounding air. The latter is generically turbulent [9], and as such is characterized by scaleinvariant velocity and energy distributions. Thus it appears more promising to seek the explanation for power-law distributions in atmospheric fluid dynamics.

\section{Chaotic Advection}

In this and the following sections we will be interested in the dynamics of passive tracer particles in a fluid. Such a particle follows the local velocity of the fluid at each moment, so that its trajectory is that of a fluid particle. The fluid velocity is not affected by the tracers. As such, a tracer represents an idealized limiting case of a very small, neutrally buoyant particle immersed in the fluid. (Tracers are small in the sense that (1) their inertia is negligible and (2) the fluid velocity varies little over the diameter of the tracer.) The idea of the model to be developed below is that raindrops can be treated, to a first approximation, as passive tracers, even though they are much denser than air, and not always "small." This study should nevertheless provide a preliminary indication of how atmospheric motion can affect the distribution of the raindrops.

Now, if the fluid motion is turbulent, the distribution of passive tracer particles should also exhibit scale-invariant properties $[12,15,16]$. An important example is Richardson's law, the empirical result that in turbulent flow, the mean-square separation $\ell_{t}$ between a pair of tracers at time $t$, given an initial separation of $\ell_{0}$, grows $\sim \ell_{0}^{4 / 3}$. If two or more tracers are released at nearby points, we can study how their trajectories separate over time, leading to the notion of chaotic tracer motion: trajectories that separate exponentially rapidly with time. A flow need not be turbulent to exhibit chaos in this sense. Relatively simple flows, such as the van Karman vortex street or flows generated by systems of point vortices exhibit this property. Aref showed that this phenomenon, known as chaotic advection or Lagrangian chaos, appears in systems of as few as four mutually interacting vortices $[13,14]$. (The vortex system, which is central to the model developed here, will be described in detail below.)

Some aspects of chaotic advection can be understood in a general way using elementary notions from dynamical systems theory. Consider an incompressible fluid restricted to a finite volume. A stagnation point in such a flow is a hyperbolic fixed point: due to volume conservation, the fluid is attracted to this point along one direction, and repelled along another. As a result, a fluid element that passes near the hyperbolic point is stretched along one direction, compressed along the other. As stretching continues, the element must double back on itself since it is confined to a finite region. Repeated encounters with hyperbolic points lead to iterated distortions of the kind described above. Thus a fluid element undergoes repeated stretching and folding similar to the distortions leading to chaos in simple model systems such as the baker's transformation [35].

Flow fields with chaotic advection may also exhibit unstable periodic orbits with fractal structure[11]; tracers (as well as particles with non-negligible inertia) may spend long periods of time in the vicinity of these orbits [36]. The effect, once again, is that an initially compact region becomes highly extended along one direction, and contracted in the other, and repeatedly folded, yielding a self-similar structure of bands reminiscent of a strange attractor in a chaotic dynamical system.

Summarizing, the motion of tracer particles in even moderately complex flows can yield chaotic trajectories and scale-invariant spatial distributions. This suggests treating rain as a collection of passive tracers moving in a chaotic or turbulent velocity field. The raindrops are released in a localized condensation event, and then advected by the air before being detected at or above a given point on Earth's surface.

What would a reasonably complete model of this process look like? Even ignoring thermodynamic aspects (evaporation and re-condensation of rain, with attendant latentheat and buoyancy effects), we would need to treat a threedimensional atmosphere whose density falls off exponentially with height, and integrate the Navier-Stokes equation for an incompressible fluid subject to suitable boundary and initial conditions, (including a driving term at large scales to compensate small-scale dissipation, if we wish to study a stationary state), at a Reynolds number characteristic of turbulent motion [37]. To include the possibility of convection we would need to implement (at least) the Boussinesq approximation, allowing the density to vary linearly with temperature, and including heat transfer in the description $[38,39]$. Such a study poses a great challenge to presently avaliable computational tools. In particular, faithful representation of fully developed turbulence appears (due to the number of degrees of freedom involved) computationally nonviable, so that reduced descriptions such as large-eddy simulation or a shell model are required [7, 8].

While semi-realistic simulation seems a worthy objective for future study, in this work I consider a radically simplified model, which can serve as a proof of principle of the idea that fractal rain distributions derive from chaotic advection. The model eliminates nearly all atmospheric processes and takes advantage of a physical system (point vortices) affording a vast reduction in computational complexity, as explained in the next section.

\section{Computational Model}

On the planetary scale, Earth's atmosphere is twodimensional. At high Reynolds numbers, effects of viscosity are limited to small scales and to boundary layers. These observations may be seen as possible motivations for what is in the final analysis a simplification based on computational necessity, namely, the study of ideal two-dimensional flow. In potential flow, i.e., for which the fluid velocity $\mathbf{u}(\mathbf{x}, t)$ can be written as the gradient of a scalar function $\phi(\mathbf{x}, t)$ $[40,41,42]$, the incompressibility condition $\nabla \cdot \mathbf{u}=0$ implies that $\phi$ satisfies Laplace's equation; such flows are irro- 
tational, i.e., $\nabla \times \mathbf{u}=0$. Potential flow solutions of Euler's equation satisfy the principle of linear superposition.

The velocity field is built up out of complex potentials of the form

$$
\phi=-i \frac{K}{2 \pi} \ln (x+i y)
$$

corresponding to the velocity field (in polar coordinates)

$$
u_{\theta}=\frac{K}{2 \pi r}, \quad u_{r}=0 .
$$

(The circulation $K$ is the line integral of the velocity over any circuit including the origin; $\nabla \times \mathbf{u}=0$ except at the origin, where the velocity is evidently singular.) We construct more complicated flows by superposing vortices at different points $\mathbf{r}_{j}$. (The vortex is an extended object; $\mathbf{r}_{j}$ denotes the position of the singularity.) In a system of $N_{V}$ point vortices, each vortex $j$ moves in the velocity field defined by the superposition of all vortices except vortex $j$ itself [41]. (For $N_{V} \leq 3$ the system is integrable [14].) Thus, in this rather special case we can construct a complex fluid motion without solving the Euler equation, by integrating the motion of a system of $N$ point particles. This makes the vortex system particularly attractive for simulating incompressible, inviscid flow.

Point-vortex systems have been used for some time in studies of two-dimensional turbulence [7, 43, 44] and of chaotic advection $[13,14]$, and appear to be relevant to atmospheric dynamics on various scales [45]. Two interesting scaling properties of tracers in systems of four or more point vortices are worth noting [14]: (1) the tracers exhibit anomalous difusion, with the mean-square displacement growing $\sim t^{1.8}$; (2) the lifetime $s$ of vortex pairs follows a powerlaw distribution, $P(s) \sim s^{-2.7}$. (Tracers are typically excluded from the immediate vicinity of a vortex, but may on the other hand become trapped at the periphery of a vortex pair.) Compared with direct integration of the Euler or Navier-Stokes equations, the computational demands are orders of magnitude smaller. Of course, one is restricted to a two-dimensional, inviscid fluid. (In the three-dimensional case the vortices become vortex lines, which stretch and fold under the flow. But such a system may still offer computational advantages.)

In Ref. [17] I study a system of interacting point vortices on the unit square with periodic boundaries. The velocity of vortex $i$ is given by

$$
\mathbf{v}_{i}=\sum_{j \neq i} \frac{K_{j}}{2 \pi r_{i j}^{2}} \hat{\mathbf{k}} \times \mathbf{r}_{i j},
$$

where $K_{j}$ represents the circulation of vortex $j$ (equal numbers of clockwise and anticlockwise vortices are used), and $\mathbf{r}_{i j}=\mathbf{r}_{i}-\mathbf{r}_{j}$, under periodic boundaries, using the nearestimage criterion. The velocity $\mathbf{u}(\mathbf{x}, t)$ at an arbitrary point $\mathbf{x}$ in the plane (not occupied by a vortex) is given by a similar sum including contributions from all vortices. The number of vortices $N_{V}$ ranges from 10 to 126 .

Several types of vortex-strength distributions are studied; the simplest assigns all vortices the same strength $|K|$. Other studies employ a hierarchical vortex distribution, defined as follows. The zeroth "generation" consists of a pair of vortices with $K= \pm K_{0}$. Subsequent generations, $n=$ $1, \ldots, g$ have $2^{n+1}$ vortices, with circulation $|K|=K_{0} / \alpha^{n}$. I study $\alpha=2,3$, and 4 , using $g+1=5$ or 6 generations. The purpose of the hierarchical distribution is to provide structure on a variety of length scales, without trying to reproduce any specific energy spectrum $E(k)$. The vortices are assigned random initial positions, but their subsequent evolution is deterministic [46].

Being point objects, the vortices possess no intrinsic length scale. (Note however that in the presence of other vortices, the 'sphere of influence' of vortex $i$ is proportional to $K_{i}$.) A characteristic length scale is the mean separation $\sim 1 / \sqrt{N_{V}}$ between vortices. The vortex system defines a mean speed $u=\langle|\mathbf{u}(\mathbf{x}, t)|\rangle \propto K_{0} \sqrt{N_{V}}$; an important time scale is $\tau_{C} \sim 1 / u$, the typical time for a fluid particle to traverse the system. A typical velocity field in a system of ten vortices (all of equal intensity) is shown in Fig. 1.

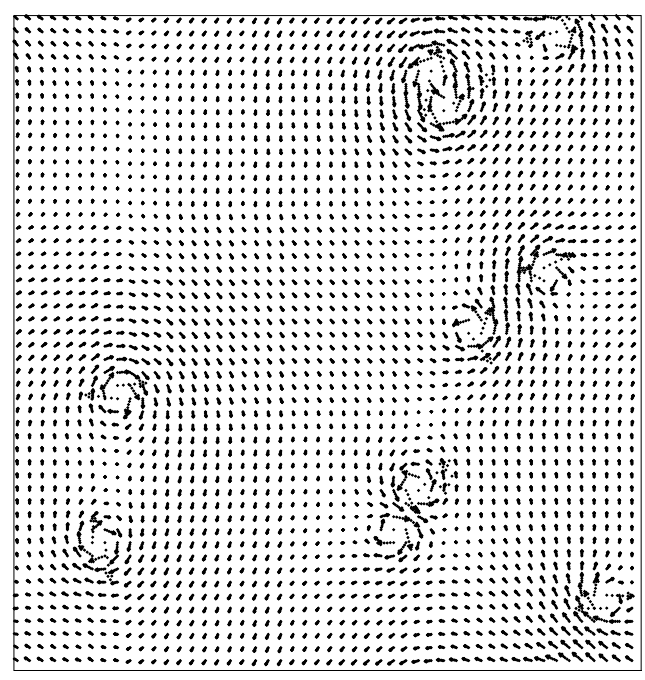

Figure 1. Velocity field in a system of ten vortices of equal strength.

A large number of tracers, $N_{p}=10000$, are thrown at random into a small region (a square of side 0.05), representing a localized condensation event. (Alternatively, the tracer-laden region may be interpretated as a parcel of atmosphere of high humidity, destined to generate precipitation.) In the analysis of rain and drought events, the observation interval $T$ plays an important role. At time zero the vortices begin their motion, and the tracers are inserted. The dynamics is followed up to time $T$, when the simulation ends.

In the model, 'rain' corresponds to the presence of one or more tracers in a very small predefined region or "weather station', of linear dimension 0.01. At each step of the integration, the number of particles $n_{i}(t)$ at each station $i$ is monitored. A sequence of nonzero occupation numbers at a given station constitutes a rain event, just as in the radar observations [4]; the intensity of a rain event is $I=\sum_{t} n_{i}(t)$ where the sum is over the set of consecutive time steps for 
which $n_{i}(t)>0$. In case $n_{i}=0$, station $i$ is said to experience a drought. The durations of droughts and of rain events are likewise monitored over a time interval $T$.
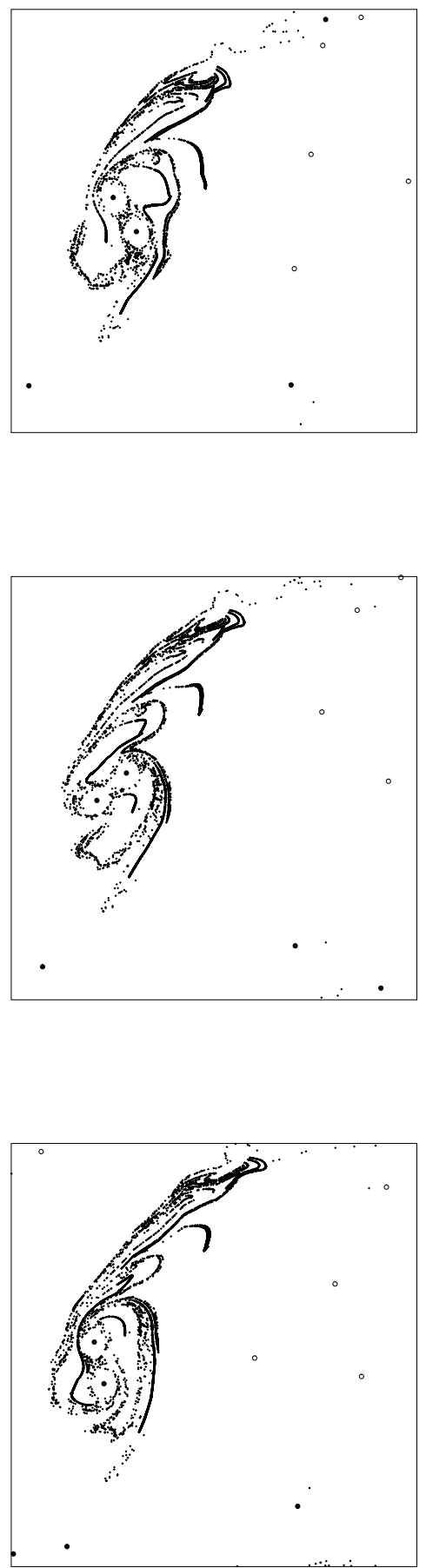

Figure 2. Positions of $10^{4}$ tracers (small points) and 10 vortices (open circles, clockwise circulation, filled, anticlockwise), at times 0.46 (a), 0.48 (b) and 0.50 (c).

Fig. 2 shows successive configurations of a system of $10^{4}$ particles and 10 vortices of equal intensity, at times 0.46 , 0.48 and 0.50 , under conditions such that $\langle|\mathbf{u}(\mathbf{x}, t)|\rangle=4$.
(Thus $T=0.5$ corresponds to $2 \tau_{C}$.) In this example the tracer-bearing region has become wrapped around a vortex pair, and becomes increasingly stretched. The tracers are widely scattered, but their distribution remains highly nonuniform, characterized by bands of high particle concentration. (The tracer-free regions centered on the vortex pair arise because the fluid trajectories circulate about the vortices, so that tracers cannot penetrate this region from outside.) At later times (see Fig. 3, for $T=8 \tau_{C}$ ) tracers are more uniformly distributed, but there are again empty regions centered on vortices or vortex pairs. (In these studies the tracers were released from a region of size $0.01 \times 0.01$ to provide enhanced spatial resolution.)

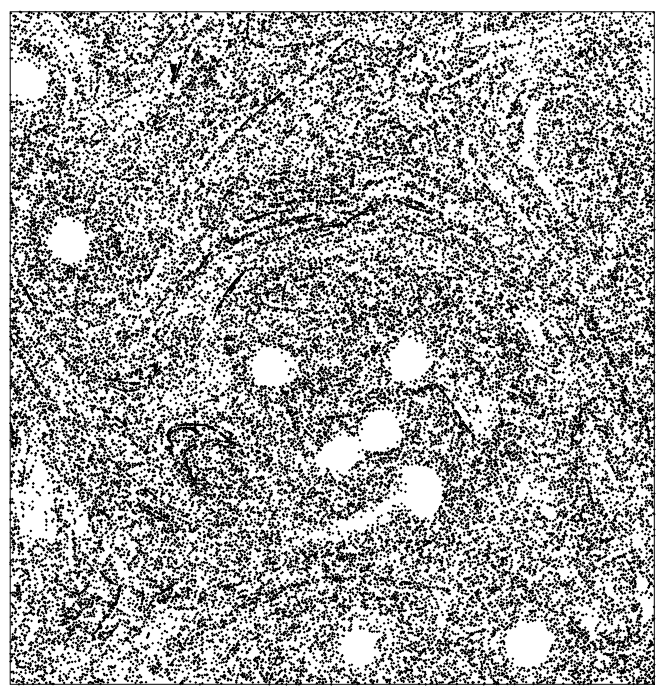

Figure 3. Positions of $5 \times 10^{4}$ tracers at time $8 \tau_{C}$, for the same conditions as Fig. 2.

Varying the vortex distribution and observation interval $T$, the following trends emerge. For $T / \tau_{C}$ in the range 0.1 - 2, power-law rain-intensity and drought duration distributions are found, as in Eqs. (1) and (2). The rain-intensity distribution follows a power law over 4 - $51 / 2$ decades, with an exponent $\tau_{I}$ in the range $0.93-1.02$. The drought-duration distribution decays with a somewhat larger exponent, 1.12 1.16, and follows a power law over 3 - 4 decades. Larger exponent values are associated with higher values of $\alpha$; these yield somewhat smaller ranges for the power laws. Conversely, the largest power-law range, and smallest exponent values, are observed when all vortices are of equal strength. There is no significant difference between the distributions obtained initially and those found after the vortices have had some time to evolve, suggesting that the equilibration process expected in two-dimensional turbulence [43, 44] is not important as regards rain and drought statistics.

Systems with varying numbers of vortices yield similar distributions, if we scale the intensity $K \sim 1 / \sqrt{N_{V}}$. This is seen from the data collapse in Fig. 4, in which results for systems of $10,20,50$ and 100 vortices (with $N_{p}=10^{4}$, $T \simeq 0.85 \tau_{c}$, and $K=0.3$ for $N_{V}=10$ ), are shown. Even systems with as few as ten vortices yield good power laws, indicating that chaotic advection is the essential feature leading to scale invariance, rather than well developed 
turbulence.

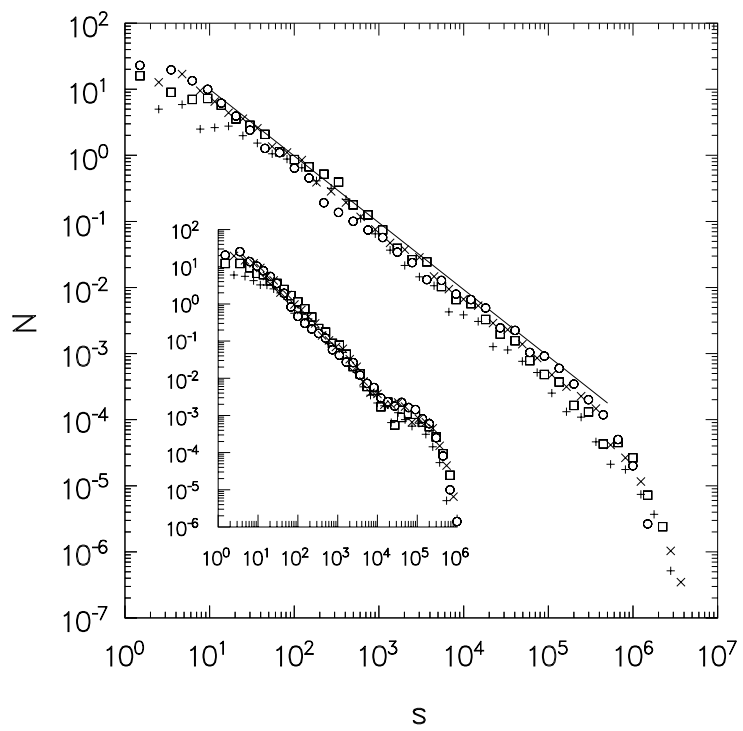

Figure 4. Rain-size (main graph) and drought-duration (inset) distributions in systems of vortices of equal strength, $T \simeq 0.85 \tau_{c}$. ०: $N_{V}=10 ; \times: N_{V}=20 ; \square: N_{V}=50 ;+: N_{V}=100$. The vortex intensity $K$ is scaled $\sim 1 / \sqrt{N_{V}}$ in these studies. The straight lines have slopes of -1.01 (rain size) and -1.13 (drought).

For larger values of $T / \tau_{C}$ the particles are more dispersed, and the rain size and drought duration follow a stretched-exponential form $P_{i}(I) \propto \exp \left(-C I^{\beta}\right)$ with $C$ a constant and $\beta \simeq 0.5$. Even for large values of $T / \tau_{C}$ (up to 200 in the present study), the distributions decay more slowly than an exponential, showing that the tracer density is non-Poissonian.

The results of [17] may be summarized as showing scale-invariant rain-size and drought-duration distributions for intervals such that the tracers remain highly clustered. Although the decay exponents are somewhat smaller than those obtained from observational data (1.36 and 1.42 for rain size and drought duration, resp. [4]), the simulations also show the drought duration decaying more rapidly than that for rain event sizes. For conditions under which the rain is more thoroughly dispersed, simulations yield stretchedexponential distributions. It is worth noting that the finding of non-power-law distributions at longer times does not signal an inability of the model to reproduce the observational results. Rain, after all, does not remain in the air indefinitely. (It would, of course, be interesting to have some way of comparing the model timescale $\tau_{C}$ with the typical residence time of rain in the atmosphere.) The tendency toward a more uniform tracer distribution at times $\gg \tau_{C}$ is in fact exagerated by the periodic boundaries of the model, and might occur more slowly under a corresponding vortex dynamics in the atmosphere.

Even in a system as simple as that considered here, there is a large parameter space to be explored: number, circulation, and intensity of vortices, size and shape of the initial particle-bearing region, observation time $T$. To close this section I report some preliminary results on situations not considered in [17]. In all cases there are ten vortices, all of intensity 0.3 , yielding $\langle|\mathbf{u}(\mathbf{x}, t)|\rangle=4$. A study in which the tracers are released from a circular, rather than a square region yields the same exponents $\tau_{I}$ and $\tau_{D}$ as found previously. Thus the shape of the initial region appears not to influence the event statistics.

It is natural to ask how relaxing the "neutrality condition" (equal numbers of vortices with clockwise and anticlockwise circulation) affects the event distributions, since there is no obvious reason to assume such neutrality. A study using all vortices with the same circulation again yields power-law distributions, but with somewhat different exponent values, depending on the observation time. Specifically, for $T=0.8 \tau_{C}$ I find $\tau_{I}=1.01(1)$ and $\tau_{D}=1.10(2)$, similar to the results for the neutral system, while for $T=$ $1.2 \tau_{C}, \tau_{I}=1.21(1)$ and $\tau_{D}=1.06(1)$. Thus, allowing a net circulation results in a larger rain intensity exponent at longer times, while the drought exponent is slightly smaller.

There is also evidence that releasing the tracers from a smaller region (of linear size 0.01 instead of 0.05 ) yields a larger $\tau_{I}$ and smaller $\tau_{D}$. A study using $T=0.5$ (and equal numbers of clockwise and anticlockwise vortices), yielded $\tau_{I}=1.10(2)$, while $\tau_{D} \simeq 1.02$. Although it is difficult to draw firm conclusions from these preliminary results, they demonstrate the generality of power-law distributions at intermediate times, while suggesting that exponent values may change depending on the flow regime.

\section{Spatial Distribution of Tracers}

As discussed in the preceding section, a very simple model of passive tracers in a velocity field defined by a system of point vortices is capable of yielding power-law rainintensity and drought-duration distributions [17]. These results for events at a fixed observation site suggest that the spatial arrangement of the tracers is somehow related to the event distributions. One might even hope to understand the scale-invariant event distributions as arising from a fractal tracer pattern as it sweeps over the observation site. In this section I present results on the spatial distribution of the tracer particles, which can be thought of as analogous to the distribution of rain over a region experiencing storms. The results are for systems with equal numbers of clockwise and anticlockwise vortices, all of equal intensity $K$.

As a first step, I consider the occupancy histogram $N(n)$ upon partitioning the system into a fine mesh; $N(n)$ denotes the number of elements with tracer occupancy $n$. The simulation cell is divided into $10^{4}$ square regions or boxes of side 0.01 , and the box-occupancy histogram determined after allowing the particle configuration evolve for a time $T$. Recall that initially, a small number of boxes ( 25 or so) will have high occupancies, while the rest are empty. If the particles tend toward a uniform distribution, we should expect the histogram to approach a Poisson distribution, with parameter 1 (there are $10^{4}$ particles) for large $T$. The simulation results instead indicate a tendency to form a power-law distribution at short times, followed by a stretched-exponential form at longer times. Fig. 5 shows histograms at various observation times for a system of 10 vortices of equal intensity, $|K|=0.3$. At times $T / \tau_{c}=0.2$ and 0.4 , a peak 
near occupancy $n=400$ is evident, a remnant of the initially compact distribution. The histogram follows a power law $N(n) \sim n^{-\epsilon}$, for $n \leq 200$ or so, with $\epsilon=0.54(1)$ for $T=0.2 \tau_{C}$. As $T$ increases, the exponent $\epsilon$ becomes larger, and the histogram (on log scales) begins to curve downward, signaling a faster than power-law decay. For $T=0.5$ the histogram is well described by a stretched exponential, $N(n) \propto \exp \left[-\right.$ const. $\left.\times x^{\beta}\right]$ with $\beta \simeq 1 / 7$. Thus the histogram remains non-Poissonian even for rather long times. For a system of 100 vortices (with $K$ scaled to maintain the mean velocity constant as discussed in Sec. V), the histogram is power law (with $\epsilon=0.67$ ) for $T=0.2 \tau_{C}$, and tends to a stretched exponential (with $\beta \simeq 1 / 5$ ) for longer times.

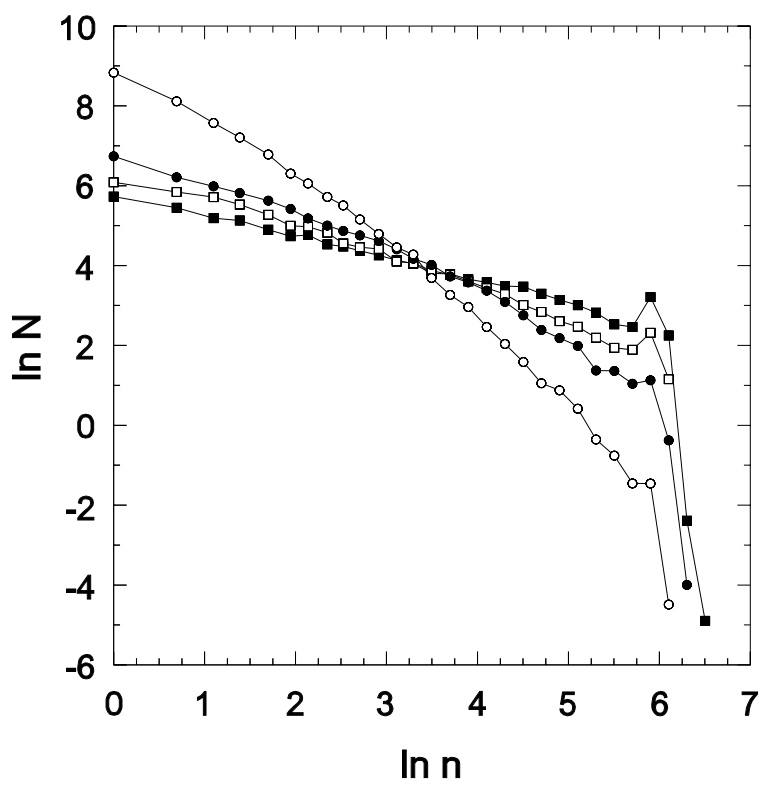

Figure 5. Instantaneous occupancy histogram $N(n)$ for boxes of side 0.01 in a system with 10 vortices, $|K|=0.3$. Observation times: $T=0.2 \tau_{c}$ (filled squares); $T=0.4 \tau_{c}(\square) ; T=0.8 \tau_{c}(\bullet)$; ०: $T=2 \tau_{c}($ ()).

In principle, the fractal dimension of the instantaneous particle distribution may be determined in a manner analogous to the fractal time distribution described in Sec. III. That is, we divide the system into ever-finer partitions (for example, squares of side $\ell=2^{-n}$ for $n=1,2,3, \ldots$ ) and determine the number $r(\ell)$ of occupied squares at scale $\ell$. For uncorrelated positions we expect $r(\ell) \propto \ell^{-2}$ away from the limits of very large or very small boxes. Applying this analysis to tracer configurations in the vortex system yields $r(\ell) \propto \ell^{-\gamma}$ with $\gamma=1.8-1.9$, depending on the observation time $T$ and number of vortices. This may signal an incipient fractal distribution, but a glance at a typical configuration (Fig. 2) shows that at the times of interest, the particle-filled region is not a fully developed fractal structure, but rather is essentially linear, becoming increasingly stretched (and wound about one or more vortices), and folded as times goes on.

The observation of a stretched, linear tracer-bearing region suggests that we distinguish two directions, locally parallel and perpendicular to the elongated region. Observe that the particle velocity is approximately parallel to the elongated direction. Thus it is of interest to define coordinates $\eta$ and $\xi$ at any instant, representing the distance from a given particle $i$ in directions parallel and perpendicular to (respectively) its velocity $\mathbf{v}_{i}$. We study the tracer density as a function of distance from a randomly chosen particle, along these directions, effectively defining two-point correlation functions $C_{\|}(x)$ and $C_{\perp}(x)$. Fig. 6 shows that at time $2 \tau_{C}$ these functions are strongly peaked near the origin, demonstrating a high degree of clustering, and that $C_{\|}(x)$ is generally greater than $C_{\perp}(x)$, corresponding to the elongated linear regions typical of the particle configuration at intermediate times. The correlation functions at time $8 \tau_{C}$ (shown in the inset of Fig. 6) are much more uniform, away from the central peak, and appear to be isotropic.

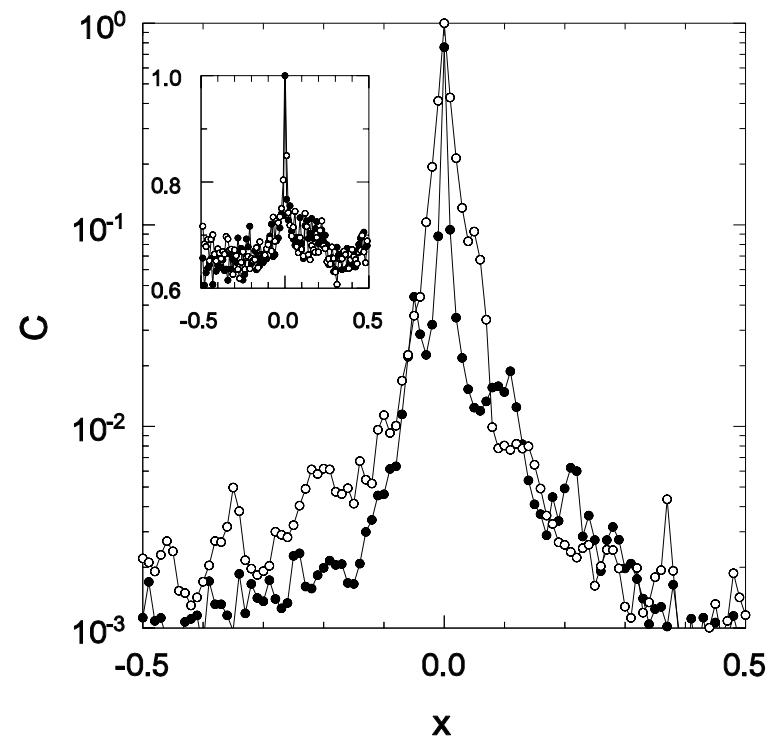

Figure 6. Correlation functions (unnormalized) $C_{\|}(x)(\circ)$ and $C_{\perp}(x)(\bullet)$ on semi-log scales, in a system with ten vortices, observation time $T=2 \tau_{C}$. Inset: a similar plot on linear scales for $T=8 \tau_{C}$.

The configurations depicted in Fig. 2 suggest that the repeated bands of particles (due to folding and/or wrapping around a vortex) possess a nested structure. We look for evidence of fractal structure along the perpendicular direction $\xi$ by dividing this axis (along a narrow swath, $|\eta| \leq 0.005$ ) into segments of length $\ell=2^{-n}$, and determining the number of occupied segments $r(\ell)$ at scale $\ell$. This function is shown for various observation times in Fig. 7. At the shortest times $r(\ell)$ is constant for larger $\ell$, indicating that only a single box is occupied at the larger scales, due to the small insertion region (of length 0.01 here). At intermediate times there is evidence of fractal scaling (for example, at $T=2 \tau_{C}$, $r \sim \ell^{-0.72}$ for $\ell \leq 0.05$ ). The slope $\gamma$ (away from the saturation region at small $\ell$ ) appears to approach unity at larger times, again signaling a more uniform tracer distribution. (For $T=2 \tau_{C}$ the distribution $r(\ell)$ in the parallel direction is very similar to that for the perpendicular direction shown in Fig. 7.) 


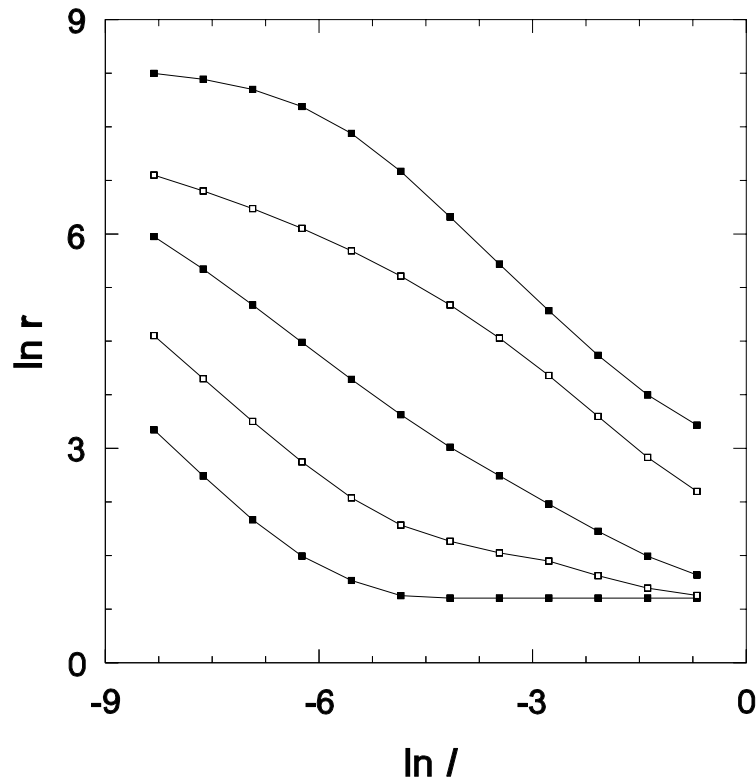

Figure 7. Distribution $r(\ell)$ along a line perpendicular to the local velocity in a system of 10 vortices. Observation times (bottom to top) $T / \tau_{C}=0.8,1.2,2.0,4.0$, and 8.0. (The data have been shifted vertically for visibility.)

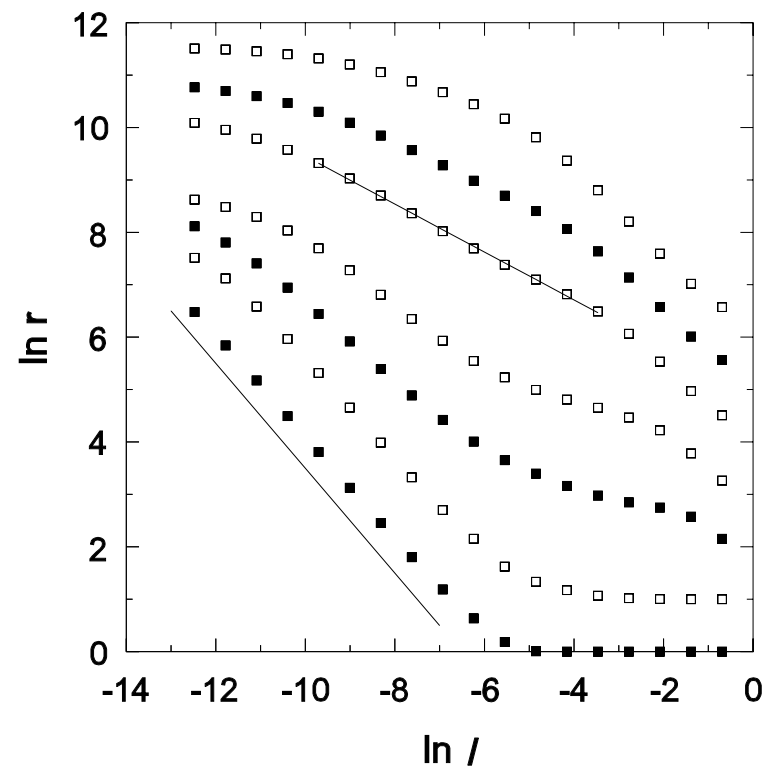

Figure 8. Distribution $r(\ell)$ as in Fig. 7, but in a single realization with $10^{5}$ tracers released from a region of size 0.005 . Observation times (bottom to top) $T / \tau_{C}=2.4,3.2,4.0,4.8,5.6,6.4$ and 8.0. (The data have been shifted vertically for visibility.) The slopes of the straight lines are -1 and -0.47 .

The results for $r(\ell)$ cited above represent averages over 5 - 10 configurations. High-resolution studies of single configurations (involving $10^{5}$ tracers released from a region of linear size 0.005), yield power-law distributions in some cases, and stretched exponentials in others, for the same parameter values. For example, in a system with ten vortices $\left(|K|=1.2, T=4 \tau_{C}\right)$, one realization yielded a stretchedexponential distribution with $\beta \simeq 0.1$, while in other cases power laws (with $\gamma=0.45-0.55$, over three or more decades), were found. The stretched-exponential appears to be associated with an overall scattering of tracers (as in Fig. 3) while in the power-law case multi-band configurations predominate. Similar results are found in a system of 20 vortices. Fig. 8 shows how the distribution evolves over time in a typical high-resolution study. At short times, $r(\ell) \sim 1 / \ell$ for small $\ell$, indicating uncorrelated positions, while at intermediate times and length scales there is evidence of power-law scaling with $\gamma \approx 0.5$, and at longer times the distribution can be fit to a stretched exponential with $\beta \simeq 0.3$.

Summarizing the results described in this section, there is preliminary evidence for a fractal tracer distribution at intermediate times (on the order of $\tau_{C}$ ) associated with the nested filamentary structures generated by stretching and folding of the particle-bearing region. The timescale for observation of a fractal tracer density corresponds roughly to that associated with fractal rain and drought distributions. (One should recall, however, that the latter are accumulated from the time the tracers are released until time $T$, whereas the tracer distributions discussed here are instantaneous.) It is easy to see that a fractal tracer distribution, swept past a fixed observation point, will generate power-law rain and drought distributions. It remains to make this connection more precise, a task complicated by the fact that the characteristics of the tracer distribution vary significantly over the observation period, and may also vary in space, as a glance at Fig. 2 suggests. This raises the possibility that the powerlaw distributions found in simulations and in actual measurements represent a superposition of distributions associated with different kinds of regions or events. It would therefore be of interest to identify simpler advection processes whose fractal properties can be determined with higher precision.

\section{Discussion}

I have reviewed observational evidence for fractal rain distributions, and discussed a highly simplified model that points to chaotic advection as the underlying reason. The detailed properties (e.g., the exponents associated with the powerlaw distributions) furnished by the model differ from those found in observations. (In truth, the present "toy" model ignores so many important atmospheric processes that quantitative agreement, if obtained, might well be regarded as fortuitous. The observational data, moreover, leave doubt as to the universality of the power laws.) Simulation of the model nevertheless leads to the significant conclusion that neither interactions between tracers (i.e., between raindrops or rain-bearing parcels of air), nor fully developed turbulence are needed to generate power-law rain and drought distributions. The model also yields stretched-exponential event distributions for longer observation times. While the latter have not been reported, it is well to recall that the observational data remain rather limited. Observations from other sites are needed to confirm the generality of power laws and the possibility of other (non-scale-invariant) forms.

Studying the occupancy statistics of boxes of various sizes, I find evidence that the tracer density evolves, under 
the vortex-system flow, to a fractal distribution at intermediate times. The nature of this distribution, and its relation to the power laws found for rain and drought events, needs to be studied in greater detail.

Clearly, the model employed in this proof-of-principle study contains a minimum of atmospheric physics. A threedimensional description, allowing for stratification, convection, and vortex stretching would be desirable, as would inclusion of condensation, evaporation, and inertial effects [47]. These improvements, all of which involve significant computational complexity and expense, can be expected to alter detailed properties such as exponent values. The vortex model may readily be adapted to include some of these effects, while others will require a full analysis of the coupled Navier-Stokes and heat equations.

Since chaotic advection is an intrinsic feature of atmospheric flow, one should expect scale-invariant distributions to appear quite generally. In this regard it is interesting to note that simulations of turbulent magnetohydrodynamic processes reproduce power-law burst distributions for solar flares $[48,49]$, and that tracer patterns similar to those reported here are also found in simulations of two-dimensional barotropic turbulence [50]. Although (in the interest of simplicity) a closed model is analyzed in this work, we should expect the same phenomenon to appear in an open model with driving and dissipation [43], due once again to the chaotic nature of tracer motion.

In summary, I find that tracer distributions in twodimensional flow, represented by a system of point vortices, exhibit scale invariance during the early stage of the dispersal process. The event distributions are associated with fractal tracer distributions in space, produced by repeated stretching and folding of fluid elemnts. It therefore seems worthwhile to develop more realistic models, to understand the observations in greater detail. Theoretical prediction of the rain and drought distributions from a model velocity field remains as a formidable challenge.

\section{Acknowledgments}

I thank Kim Christensen, Miguel A. Muñoz, Oscar N. Mesquita, Ole Peters, Guilherme J. M. Garcia, Maya Paczuski and Francisco F. Araujo Jr. for helpful discussions. This work was supported by CNPq, and CAPES, Brazil.

\section{References}

[1] S. Lovejoy and B. Mandelbrot, Tellus 37A, 209 (1985).

[2] J. Olsson, J. Niermczynowicz, and R. Berndtsson, J. Geophys. Res. 98 (D12), 23265 (1993).

[3] J. Lavergnat and P. Golé, J. Appl. Meteor. 37, 805 (1998).

[4] O. Peters, C. Hertlein, and K. Christensen, Phys. Rev. Lett. 88, 018701 (2002).

[5] O. Peters and K. Christensen, Phys. Rev. E 66, 036120 (2002).

[6] P. Bak, C. Tang, and K. Wiesenfeld, Phys. Rev. Lett. 59, 381 (1987).
[7] W. D. McComb, The Physics of Fluid Turbulence (Oxford University Press, Oxford, 1990).

[8] U. Frisch, Turbulence: The Legacy of A. N. Kolmogorov (Cambridge University Press, Cambridge, 1995).

[9] S. Lovejoy, D. Schertzer, and J. D. Stanway, Phys. Rev. Lett. 86, 5200 (2001).

[10] D. J. Tritton, Physical Fluid Dynamics (Oxford University Press, Oxford, 1988).

[11] Z. Toroczkai, G. Károlyi, A. Péntek, T. Tél, and C. Grebogi, Phys. Rev. Lett. 80, 500 (1998); G. Károlyi, A. Péntek, Z. Toroczkai, T. Tél, and C. Grebogi, Phys. Rev. E 59, 5468 (1999).

[12] T. Elperin et al., Phys. Rev. E 66, 036302 (2002).

[13] H. Aref, J. Fluid Mech. 143, 1 (1984).

[14] X. Leoncini and G. M. Zaslavsky, Phys. Rev. E 65, 046216 (2002).

[15] See Chs. 12 and 13 of [7] and references therein.

[16] G. Falkovich, K. Gawȩdzki, and M. Vergassola, Rev. Mod. Phys. 73, 913 (2001).

[17] R. Dickman, Phys. Rev. Lett. 90, 108701 (2003).

[18] S. Lovejoy, M. Lilley, N. Desaulnies-Soucy, and D. Schertzer, Phys. Rev. E 68, 025301(R) (2003).

[19] See http://w3.gkss.de/baltex/ for information on the BALTEX project.

[20] The literature on fractal rain distributions is extensive; for a bibliography see [18].

[21] B. Gutenberg and C. F. Richter, Bull. Seismol. Soc. Amer. 34, 185 (1944).

[22] F. Omori, J. College Sci. Imper. Univ. Tokyo 7, 111 (1895).

[23] K. Christensen, L. Danon, T. Scanlon, and P. Bak, Proc. Nat. Acad. Sci. 99, suppl. 1, 2509 (2002).

[24] R. Burridge and L. Knopoff, Bull. Seismol. Soc. Am. 57, 3411 (1967).

[25] J. M. Carlson, J. S. Langer, and B. E. Shaw, Rev. Mod. Phys. 66, 657 (1994).

[26] R. Dickman, M. A. Muñoz, A. Vespignani, and S. Zapperi, Braz. J. Phys. 30, (2000) 27.

[27] R. Frigg, Stud. Hist. Phil. Soc. 34, 613 (2003).

[28] S. T. R. Pinho and R. F. S. Andrade, Physica 255A, 483 (1998).

[29] R. F. S. Andrade, S. T. R. Pinho, S. C. Fraga, and A. P. M. Tanajur, Physica 314A, 405 (2002).

[30] The sandpile model studied in [28] involves rather specific assumptions regarding stability, breakup and coalescence of raindrops. Moreover, it yields non-power-law distributions for rain event sizes and interevent durations. It does however yield a power-law distribution for "internal avalanches" (those not leading to rainfall) with an exponent $\tau \simeq 4 / 3$, rather close to that found by Peters et al.

[31] G. Grinstein, in Scale Invariance, Interfaces and Nonequilibrium Dynamics, NATO Advanced Study Institute, Series B: Physics, vol. 344, A. McKane et al., Eds. (Plenum, New York, 1995). 
[32] A. Vespignani and S. Zapperi, Phys. Rev. Lett. 78, 4793 (1997); Phys. Rev. E 57, 6345 (1998).

[33] E. T. Lu, Phys. Rev. Lett. 74, 2511 (1995).

[34] M. J. Manton, Rep. Prog. Phys. 46, 1393 (1983).

[35] J. M. Ottino, The Kinematics of Mixing: Stretching, Chaos, and Transport, (Cambridge University Press, Cambridge, 1989).

[36] C. Jung, T. Tél, and E. Ziemniak, Chaos 3, 555 (1993).

[37] The incompressibility condition should be well satisfied since the velocities of interest are small compared to the speed of sound.

[38] S. Chandrasekhar, Hydrodynamic and Hydromagnetic Stability (Clarendon Press, Oxford, 1961).

[39] P. Manneville, Dissipative Structures and Weak Turbulence (Academic Press, New York, 1990).

[40] D. Landau and E. M. Lifshitz, Fluid Mechanics, (Pergamon Press, Oxford, 1959).

[41] A. Sommerfeld, Mechanics of Deformable Bodies (Academic Press, New York, 1950).

[42] Further details and extensive references on systems of point vortices may be found in Ref. [14].
[43] H. Aref and E. D. Siggia, J. Fluid Mech. 100, 705 (1980); E. D. Siggia and H. Aref, Phys. Fluids 24, 171 (1981).

[44] R. H. Kraichnan and D. Montgomery, Rep. Prog. Phys. 43, 35 (1980); R. H. Kraichnan, Phys. Fluids 10, 1417 (1967).

[45] D. G. Dritschel and B. Legras, Phys. Today, March, 1993, p. 44 , and references therein.

[46] It would be of interest to study other initial configurations, for example, one approximating a vortex sheet, which should then suffer a Kelvin-Helmholtz instability. The periodic boundaries might also be removed, allowing the vortex system to attain its natural, unconstrained size.

[47] I. J. Benczik, Z. Toroczkai, and T. Tél, Phys. Rev. Lett 89, 164501 (2002).

[48] G. Boffetta et al., Phys. Rev. Lett 83, 4662 (1999).

[49] Scaling properties of solar magnetic bursts have also been studied in the SOC context. See D. Hughes et al., Phys. Rev. Lett 90, 131101 (2003).

[50] G. Haller and G. Yuan, Physica D 147, 352 (2000). 